\title{
Seek Difference While Reserving Common Points
}

\author{
Yuzhou Wu \\ Huaiyin Institute of Technology \\ Huaian, China 223001
}

\author{
Ping Liu \\ Huaiyin Institute of Technology \\ Huaian, China 223001
}

\begin{abstract}
With iPhone working as the research object, this paper makes in-depth analysis and interpretation on the consistency and difference in its appearance, details, color and material and others. On the basis of this, it is points out that in the process of visual image design of product, designers should seek the difference of design in order to highlight the individuality of the design while keeping the consistency of visual image.
\end{abstract}

Keywords-iPhone; design; minimalism; consistency; differences

\section{INTRODUCTION}

In this era, product features are increasingly homogeneous, which means that people more and more concern about the product's individuality and its image in their product choice of consumption. However, in the strict sense, "individuality" and "image" is a pair of antonyms, because individuality means difference, and "image" contains certain stability. Therefore, how to solve this contradiction and to organize "all the visual factors" of a brand and become them a unified body in a simple highstandard quality" has become a problem of a designer to have to solve. Apple Inc, taking design as the core competitiveness, has properly dealt with the contradiction between "image" and "individuality" in its iPhone products, which provides designers a good reference: iPhone series of mobile phones keep the same style of the product, but clearly reflect the intergenerational evolution.

Consistency means the universality, identity, and formal similarity of the entire products of series, which is rooted in common design philosophy and guarantees the iPhone's lineage, while the evolution means the differences and movement between individual products, and the differences based on similarity promote products from one form to another form, to ensure each product has its own character and the release of products are worth the wait, and also can promote Apple phone' fans to replace their phones (this can be considered a plan practice to make a abolishment), for which iPhone has also become a fashionable product. The success of IPhone product is just a typical case of perfect combination of the "consistency" with the "individuality". This paper made analysis on the visual image design of iPhone series, hoping to provide designers with some reference and inspiration.

\section{THE HUMANIST High-TECH: THE OVERALl VISUAL IMAGE OF IPHONE SERIES}

At the 2010 WWDC Conference, Steve Jobs pointed out that "Apple is not only a technology company, but also pursues technical and cultural integration". The perfect combination of technology and humanities is achieved in Apple products, the iPhone series of mobile phones is one of the outstanding representatives. On the Apple conference held in January 2007, Apple INC introduced the first generation of iPhone, followed by the continuous product release of " small change in one year, big change in two years"; as of early 2016, it has released 12 styles of product, including iPhone $2 \mathrm{~g}$, iPhone $3 \mathrm{G}$, iPhone3Gs, iPhone4, iPhone $4 \mathrm{~s}$, iPhone 5, iPhone 5s, iPhone $5 \mathrm{c}^{1}$, iPhone6, iPhone6 Plus, iPhone6s, iPhone6s Plus (see "Fig. 1"); the series of products have obvious intergenerational appearance, but they show a consistency of visual image under the guidance of the design concept of "humanities and technology combination", that is the so-called Apple style. The iPhone series well extend the concept of minimalism in Apple design ${ }^{1}$ : using the simplest basic modeling elements: circle (arc), rectangle, rounded rectangle, using the most "natural" organization type, through a symmetry, to unify the press key, socket and other visual elements (See "Fig. 2"); using exquisite details, plain materials, elegant colors, perfect process and structure to from the high-tech aesthetic characteristics of humanization.

\footnotetext{
${ }^{1}$ The whole iPhone5c series have extremely prominent individuality, the positive moulding is iPhone5, but the back shell is mixed with the characteristics of the first stage, and it is colorful and pure, with a large conflict with the color of the entire series, which is related with the sale price (\$99) of Apple INC. After introduced, the product did not get a good market reaction, so there is no follow-up product of $5 \mathrm{~s}$ in this pricing stage. Therefore, this author believes iPhone $5 \mathrm{c}$ is a special case in the Apple phone, and removed it from the entire series in the analysis.
} 


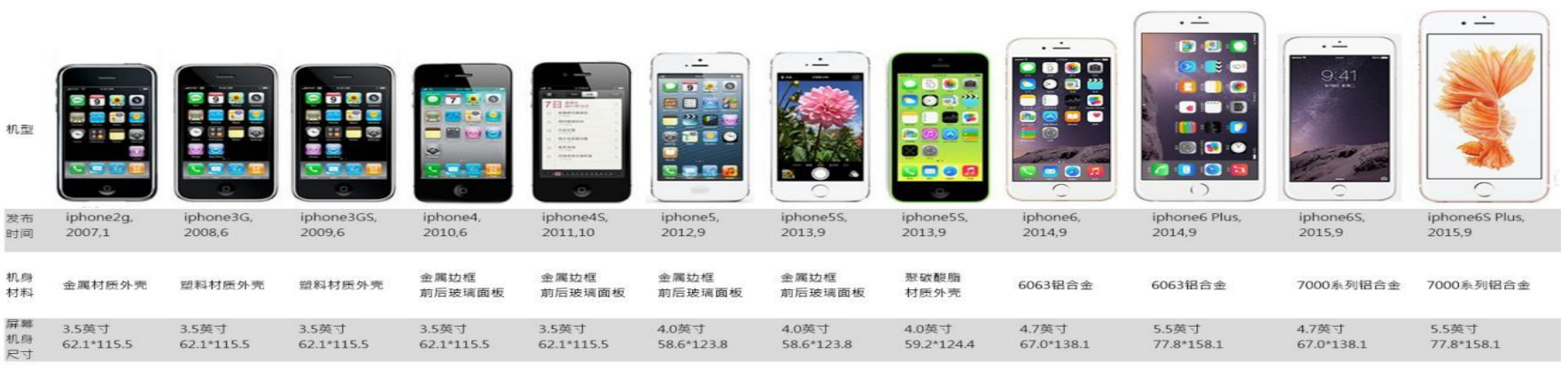

Source: from baidu pictures, self-made chart

Fig. 1. Basic situation of Apple iPhone Series

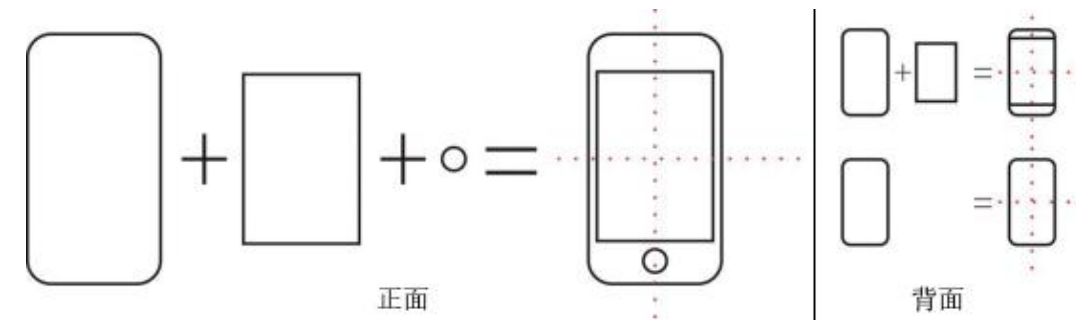

Source: self-draw

Fig. 2. The modeling elements and organization type of iPhone series

\section{THE DIFFERENCES IN CONSISTENCY: MODEL EVOLUTION OF IPHONE SERIES}

According to the philosophy of consistent design, the Apple design team made a rational use of the principle of Gestalt psychology to keep consistent treatment of the front face of the series of products as far as possible (see "Fig. 3"): the basic contour shape maintains a similar height, the delicate frame is highlighted, the visual prototype of the entire series consists of the simple rounded rectangular (radius is about $10.7 \mathrm{~mm}$ ) round home key and rectangular screen; screen and phone body are basically in axial symmetry, the receiver hole is slightly adjusted on the premise of no impact on consistency; the whole series of products show a trend of bigger and thinner one, but the size is determined basically according to the screen size, width is slightly adjusted according to the screen size, and the length is about the length of touch screen plus $20 \mathrm{~mm}$ respectively at the top and the end.
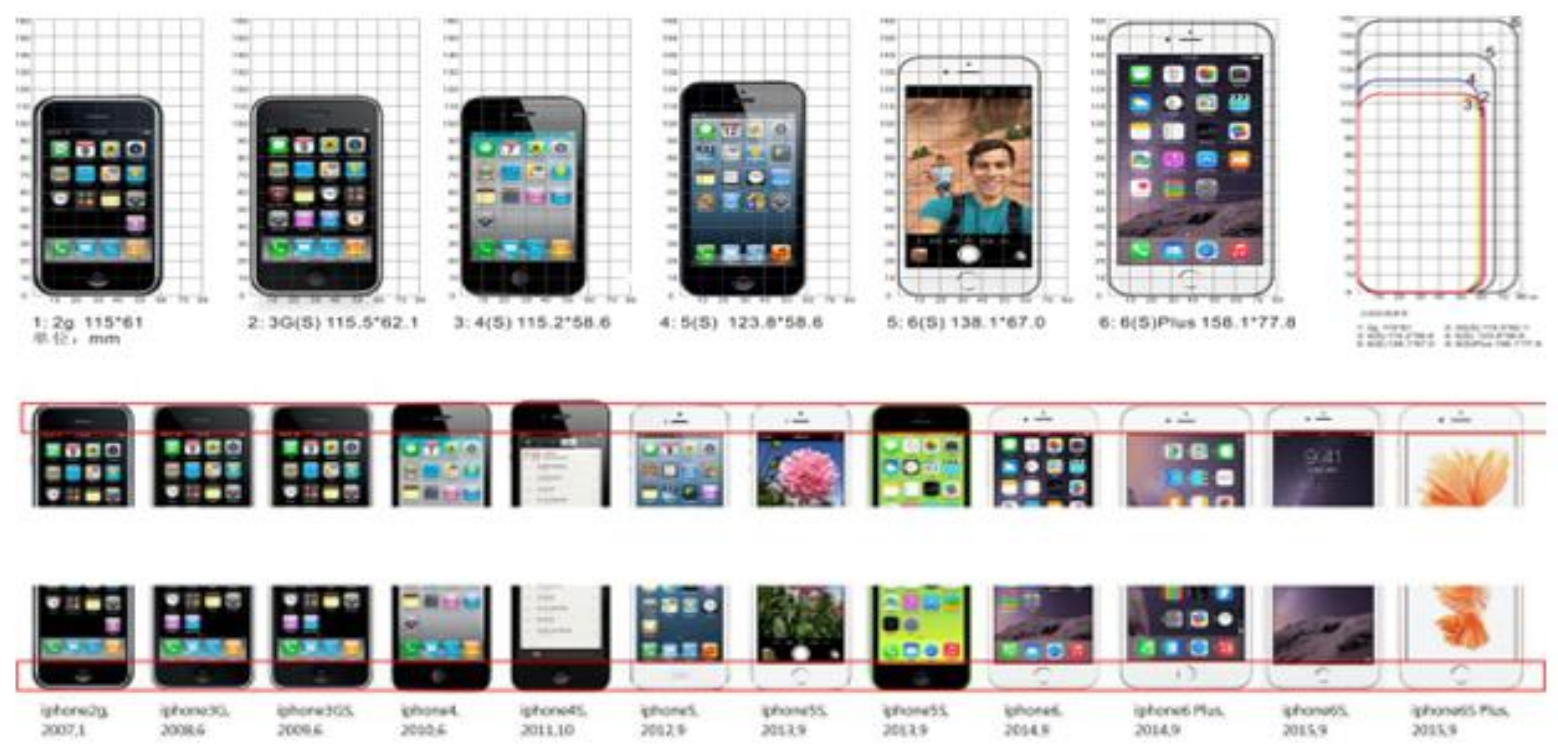

Source: From baidu pictures, self-made chart

Fig. 3. The consistency of iPhone series on the front face 
At the same time, the intergenerational differences are highlighted by the significant differences of other views. The evolution of IPhone style can be divided into three stages. (See "Fig. 4"). The first stage is for the first three generations of products (the first generation iPhone2g, the second generation iPhone $3 \mathrm{G}$, the third generation iPhone $3 \mathrm{Gs}$ ), and from the front face, the products at this stage are in axial symmetry equally up and down, left and right (this is the common layout of all Apple mobile phones), showing a rigorous and modest state. The product features at this stage depend on the "wrapped sense", and its rounded rectangular glass screen is packaged closely by smooth and full back shell, full of livingness, of which iPhone $2 \mathrm{~g}$ is the fullest, iPhone $3 \mathrm{G}$ and iPhone3Gs have a smooth and modest camber. At the second stage, it includes the four generations of products in the middle (the fourth generation iPhone4, the fifth generation iPhone4s, the sixth generation iPhone5, the seventh generation iPhone5s). Among these products at this stage, the first three generations of products have their full
3G camber replaced by plane rounded rectangle, with particularly prominent $1 / 4$ semi-circular fillet, high-tech metal frame, fine edge polishing treatment, flat design, prominent line sense of turning points at face, front and back, showing a strong sense of order and precision, and Apple's high-tech of design is particularly evident at this stage. At the third stage, it includes the rest such phones (as the eighth generation iPhone6, iPhone6 plus, the ninth generation iPhone6, iPhone6 Plus, the tenth generation iPhone7, iPhone7 plus). The products at this stage recover the modeling design of round edge (more structured compared with the first generation)and round corner before the first stage, with semi-circular metal frame, decorated with the white lines, being full, smooth and rational; the front screen and the back metal surface is wrapped very well and continuously get thinner and bigger. So, from the whole, iPhone products have a evolution trend of getting thinner and bigger in size and showing a style of "romantic and organic rationally flat - light and thin and humanist nature".

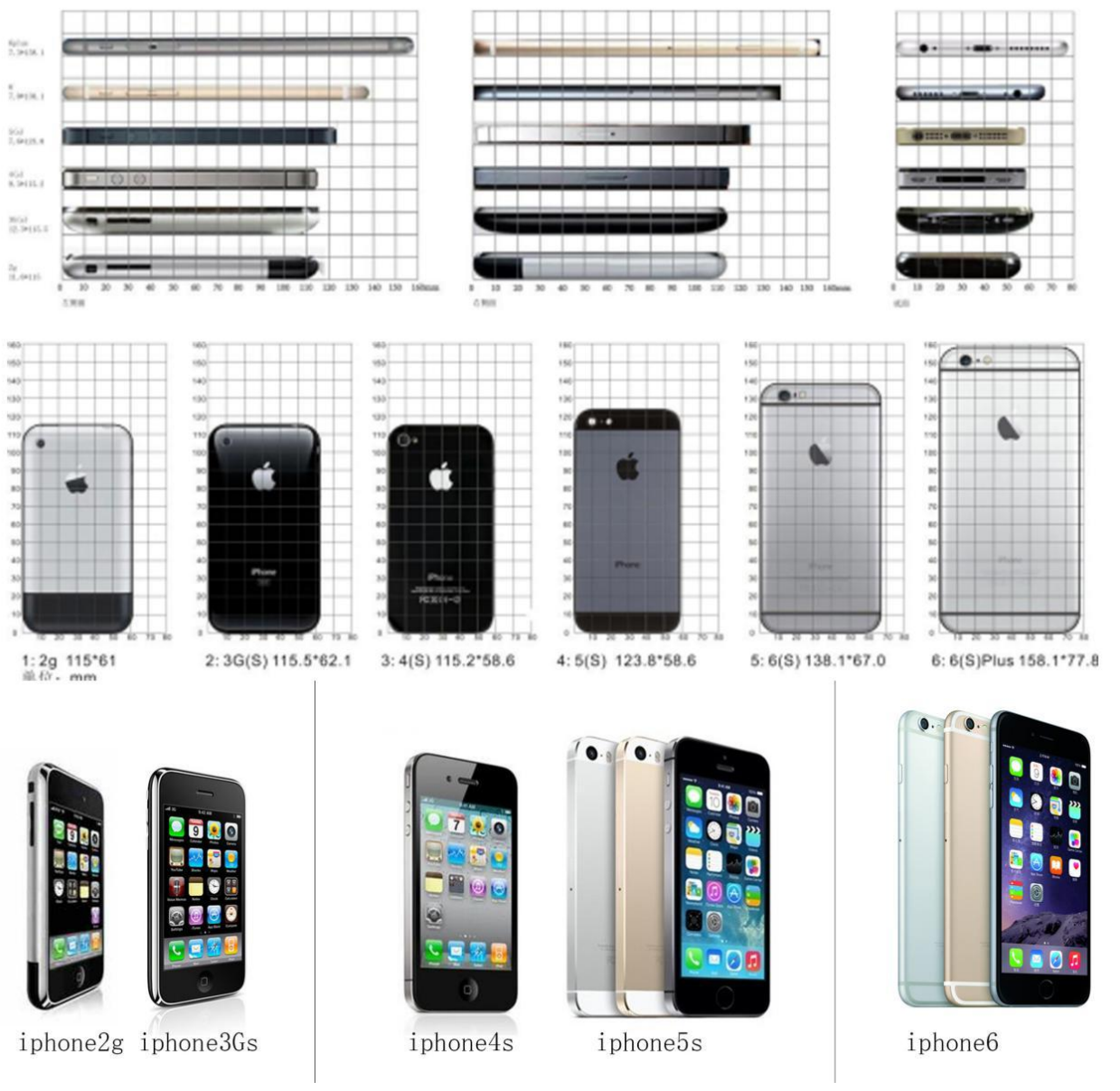

Fig. 4. The difference of iPhone series in evolution of visual style 


\section{The Changes In The COORDinAtion: Details OF IPHONE SERIES}

In the design of iPhone, all the details are considered as basic elements and are applied to the design for the sense of form. Although each model of the product has been adjusted for press keys, sockets and seams, all the details remain the consistent modeling principles: All follow the functional requirements of products (for example, in consideration of accuracy and comfort of the operation, the volume key of iPhone6 is changed from the classic round key into a long bar), and are subject to the overall layout of shape and structure (see "Fig. 5"). Under the visual guide of the design, it always focuses the people's attention on the overall feeling of the phone, and gives up highlighting some details deliberately for camping up. The entire design is unified into a simple mode of "rounded rectangle + small round" in accordance with the way of symmetry; press keys, microphone and receiver, etc. are presented directly, subject to the overall simple and elegant features, without highlighting any novel style, only for highlighting the experience of service brought about by the screen, which fully reflects Wright's thinking of " removing irrelevant items ".

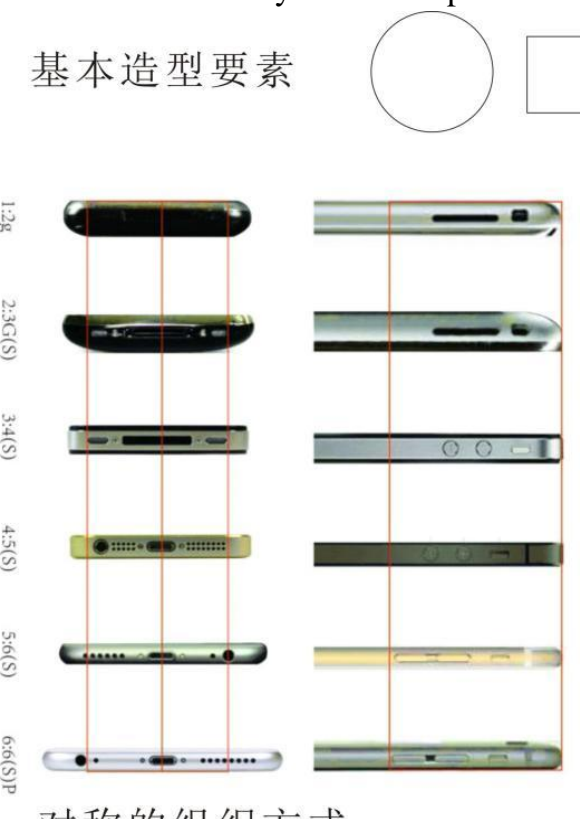

对称的组织方式

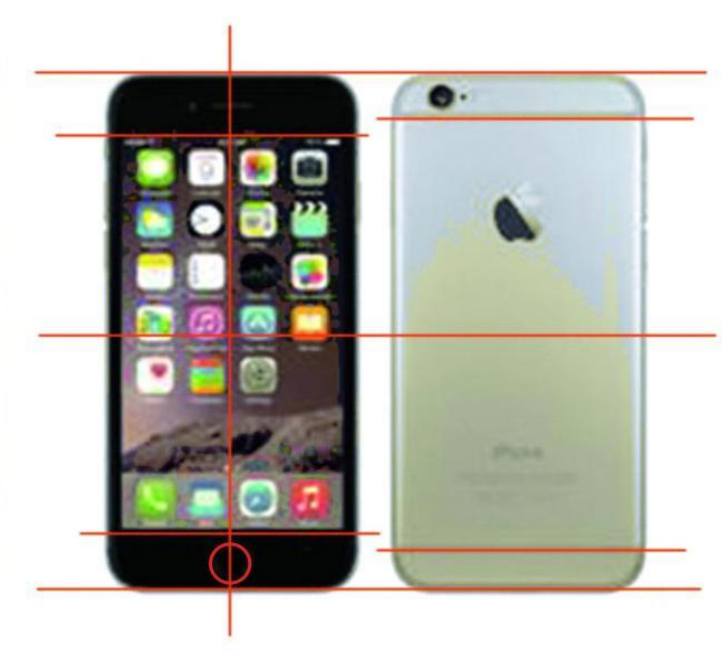

Source: from baidu pictures, self-made chart

Fig. 5. The basic modeling elements and organization type of details

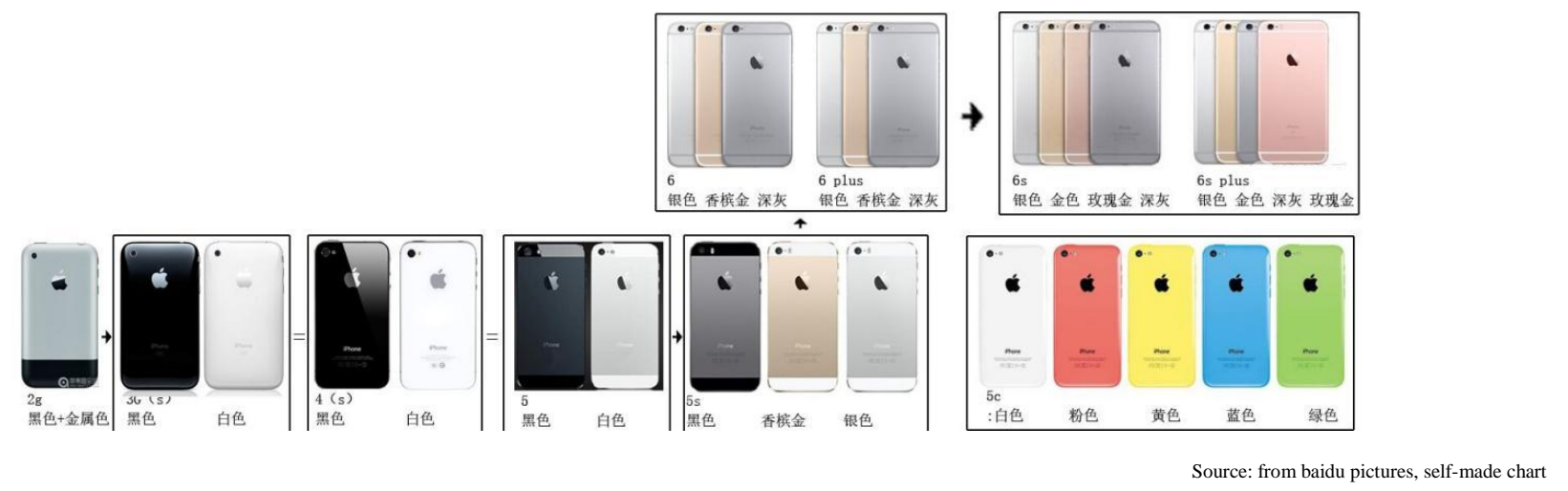

Fig. 6. Color continuation and addition of iPhone series

From the number and size, the press keys and the details are usually reduced to the limit, which is to get a sense of technology and a sense of space enough extended. Before the iPhone, smart phones usually have a layout of "screen + a large number of input keys", and the key position and fixed function occupy a larger space, resulting in failure of enlarging visual interaction screen size in the smart mobile phone, and impacting the transmission efficiency of information. For this mobile phone, objective consistency of information processing terminal is not fully achieved, so the so-called smartphone is not complete. While the Apple phone has adhered to appropriate screen size from its beginning, uses the intelligent operation of virtual keyboard and touch screen as information input of physical keyboard, 
and adopts the Home key as the assistance for control. The press keys is given multiple functions while the number of keys are reduced, to make the front face of Apple phone has only one physical key - Home key, and then the home key becomes the operation center of the entire mobile phone; the program use and exit, voice control, Phone restart, screenshots and so all can be achieved through the Home key. This simple processing is not easy: for this, designer team must make accurate adjustment and simplification for the complicacy in the application, must consider the application effectiveness of technology, materials and process, and even the usability and economy of users.

\section{FEELINGS AND AdVANTAGES : MATERIALS AND PROCESS OF IPHONE}

From the material and process, each generation of iPhone series is evolving and each generation of products are perfect. new technologies, new materials, new processes are used to bring out the best with visual image in each other, the freeness of plastic, highly cold sense of glass surface, high technology of alloy material are displayed well in each product. The refinement of the internal organization, application of uni body molding technology, improvement of surface treatment technology, use of alloy material with more strength become the means of Apple to change "and change the world" again.

According to Jonathan Ive's saying, "People feel about a design, however it is subjective, but objectively speaking, manufacturing tolerances are perceivable. We believe this, so we have moved towards these targets from the beginning of the design." for iPhone, the process of details is fine to the extreme: in the iPhone4, the metal frame has its chamfer edge less than $2 \mathrm{~mm}$ polished, with both inside and outside processed, and the black dividing line and the metal bar of the metal belt are bound tightly, without gap between them; iPhone 5 has the whole consistent modeling with iPhone 5s, but the details is more excellent, such the tiny gap in buttons of iPhone 5 is hardly found in iPhone $5 \mathrm{~S}$, the processing of aluminum frame and apple logo is finer; and iPhone6 has its size increased, and in order to keep its lightness and thinness, its frame is changed into a circular arc, and for easy operation, its on/off key is moved to the right side from the top. It can be said that this refinement of the details explains the high quality of the minimalist Apple phone due to high technology.

\section{THE CONTINUATION AND ADDITION: COLORS OF IPHONE SERIES}

The elegant color of iPhone series is very important for shaping the visual image. From the evolution of color (see "Fig. 6"), black and white is applied throughout, gold for 5s and rose gold for 6 , and limitation of this color ensures the same style. IPhone's color is pute and concise but not ordinary. In its color evolution, iPhone has always

\footnotetext{
${ }^{2}$ In the 2007 Apple conference, Steve Jobs thinks that iPhone is a unity of "music player of touch-screen control + intelligent telephone equipment + network information terminal", when he recommended iPhone as an epochmaking product.
}

maintained a pute hue, to make the texture of the material keep well in the pure luster. The same black, matched with plastic in the $2 \mathrm{~g}, 3 \mathrm{G}(\mathrm{s})$ reflects a sense of modesty, matched with glass surface in the 4 brings a sense of science and technology, matched with metal coating will evolve into a dark gray and bring a sense of elegance, this is a continuation but also a transmutation.

Color is decorative, but the colors of iPhone series have been so essential. In the definition of Steve Jobs, iPhone is the music player + intelligent communication equipment + network information terminal operated by touch-screen 2 , and its ultimate goal is to bring a better music experience, call experience and surfing experience, rather than the device itself. In the words of IVE, the complex design process is to make it seem not be designed. Therefore, the misuse of gorgeous colors or excessive decoration that transfer user's attention to iPhone itself from the service experience is inapplicable and redundant for the Apple design team who believes user first and experience supreme. In view of this, the iPhone series have no extraordinary decorative color. Therefore, the iPhone with a pure tone becomes a flexible and harmonious color place because of its frequent changes in network information, applicability app, desktop environment settings. Information is set off against this background, and this background is also vivid under the hue of the information.

\section{CONCLUSION}

IPhone series has personalized high-tech aesthetics and its visual image evolution brings us a profound inspiration: in the design of brand and serialized products, the individual characteristics and the overall consistency should be treated properly. In the modeling evolution of iPhone, the frontal (main feature view) visual feature consistency is established, to ensure the unification between the individual and the entire series, and the modeling features is reflected by changing other feature surfaces; in the overall design details, it is subject to the whole design adjustment in shape details, and adopts symmetrical organization to maintain the visual similarity of the whole series; in the processing, it adheres to excellence and perfection, and adopts reasonable materials and technology for different design needs; in color, it always maintains a simple but elegant monochrome, and assorts with different material to show different design quality, to make each generation of products similar but not the same. This method of seeking common ground while reserving differences has valuable reference for us to maintain the consistency of the visual image of products and to highlight the uniqueness of individual design, and it is worth our reference.

\section{REFERENCES}

[1] Editorial Department of Decoration: Ideas and representations minimalist classic design, "decoration", 2004,10,30.

[2] HI-ID.2010: A Design Odyssey [EB / OL].] Http://www.hiid.com/?p=2639,2010-07-12. 